Goran Tepić

Bojan Lalić

Ilija Tanackov

Siniša Sremac

Stevan Milisavljević

Milan Kostelac

https://doi.org/10.21278/TOF.43403

ISSN 1333-1124

eISSN 1849-1391

\title{
NUMERICAL MODEL OF FRAGMENTATION HAZARDS CAUSED BY A TANK EXPLOSION
}

\begin{abstract}
Summary
The paper analyses the fragmentation of a horizontal cylindrical tank caused by the effect of boiling liquid expanding vapour explosion (BLEVE). A fragmentation model for identification of kinematic parameters is proposed. The originality of the model lies in the introduction of initial acceleration. Using this model, the initial velocity can be assessed without knowing the values of explosion energy and the mass of fragments. The application of this model reduces the uncertainty in assessing the range of fragments and the risk of fragmentation. The initial acceleration of fragments generated in an explosion is assessed according to the geometry and type of the tank material. The initial acceleration, which does not depend on the kinematic parameters of the constant wall thickness of the tank, allows a reliable assessment of the launch angle of a fragment. Characteristic forms of the fragment trajectory are identified, depending on the aerodynamic and thrust acceleration coefficients, and probability distributions of the fragment ranges are given. Relevant factors in the assessment of fragmentation hazards include the trajectory of a fragment, the height of a target and its distance from the tank. It was concluded that aerodynamic fragments at distances of up to $50 \mathrm{~m}$ are not a danger to targets of up to $15 \mathrm{~m}$ high. Fragments with high air resistance and low thrust can endanger targets of the same height at distances of over $200 \mathrm{~m}$. The presented fragmentation model includes the effect of heating due to the BLEVE effect and can be applied to all types of tanks.
\end{abstract}

Key words: $\quad$ fragmentation; tank; explosion; target; hazard

\section{Introduction}

Explosions of horizontal cylinder tanks are frequent accidents in the process industry [1]. The escalation of primary accidents is manifested by cascading events known as the domino effect [2]. The initial events that most often result in the domino effect in process installations include the boiling liquid expanding vapour explosion (BLEVE) and fragmentation of the tank [3-4]. The subject of discussion presented in the paper is the 
analysis of fragmentation of the tank due to the BLEVE effect. The identification of fragmentation hazards is characterised by a high degree of uncertainty in the assessment of accident risks [5].

\begin{tabular}{|c|c|}
\hline & Nomenclature \\
\hline$D[\mathrm{~mm}]$ & External diameter of the tank $-2600 \mathrm{~mm}$ \\
\hline$h \quad[\mathrm{~mm}]$ & Height of the elliptic head $-650 \mathrm{~mm}$ \\
\hline$\delta[\mathrm{mm}]$ & Wall thickness of the tank $-14 \mathrm{~mm}$ \\
\hline$p \quad[\mathrm{bar}]$ & Operating pressure of the tank -16.7 bar \\
\hline$p_{c r}[\mathrm{bar}]$ & Critical tank pressure \\
\hline$R_{m}[\mathrm{MPa}]$ & Tensile strength of S355J2G3 steel $-490 \mathrm{MPa}$ \\
\hline$R_{e}[\mathrm{MPa}]$ & Upper yield strength of S355J2G3 steel - $355 \mathrm{MPa}$ \\
\hline$\sigma_{x}[\mathrm{MPa}]$ & Longitudinal stress of the tank \\
\hline$\sigma_{\theta}[\mathrm{MPa}]$ & Circumferential stress of the tank \\
\hline$\lambda \quad\left[\mathrm{m}^{-1}\right]$ & Coefficient \\
\hline$E[\mathrm{MPa}]$ & Modulus of elasticity \\
\hline$c \quad[\mathrm{~km} / \mathrm{s}]$ & Wave velocity \\
\hline$m_{f r}[\mathrm{~kg}]$ & Mass of a fragment \\
\hline$W_{D}[\mathrm{~N}]$ & Drag force \\
\hline$W_{L}[\mathrm{~N}]$ & Lift force \\
\hline$G \quad[\mathrm{~N}]$ & Gravitational force $-G=m_{f r} \cdot g, g=9.81 \mathrm{~m} / \mathrm{s}^{2}$ \\
\hline$\rho_{\text {air }}\left[\mathrm{kg} / \mathrm{m}^{3}\right]$ & Air density $-1.20 \mathrm{~kg} / \mathrm{m}^{3}$ \\
\hline$C_{D}\left[\mathrm{~m}^{2}\right]$ & Coefficient of drag force \\
\hline$C_{L}\left[\mathrm{~m}^{2}\right]$ & Coefficient of lift force \\
\hline$A_{D}\left[\mathrm{~m}^{2}\right]$ & Normal projection surface of a fragment \\
\hline$A_{L}\left[\mathrm{~m}^{2}\right]$ & Tangential projection surface of a fragment \\
\hline$k_{D}\left[\mathrm{~s}^{-1}\right]$ & Coefficient of drag acceleration \\
\hline$k_{L} \quad\left[\mathrm{~s}^{-1}\right]$ & Coefficient of lift acceleration \\
\hline$v_{f r}[\mathrm{~m} / \mathrm{s}]$ & Velocity of a fragment \\
\hline$a_{f r}[\mathrm{~m} / \mathrm{s}]$ & Acceleration of a fragment \\
\hline$\varepsilon_{\theta} \quad[\%]$ & Radial deformation of the tank \\
\hline$f \quad\left[\mathrm{~N} / \mathrm{mm}^{2}\right]$ & Permissible stress \\
\hline$\varphi \quad\left[{ }^{0}\right]$ & Polar angle \\
\hline$\varphi_{0} \quad\left[\begin{array}{l}0 \\
{ }^{\prime}\end{array}\right]$ & Launch angle of a fragment \\
\hline$\Delta t \quad[\mathrm{~s}]$ & Time interval \\
\hline
\end{tabular}

This fact indicates that the fragmentation analysis presented in the literature quoted above is accompanied by an insufficiently reliable estimate of the range of fragments. The purpose of this paper is to evaluate the kinematic parameters of the fragments generated due to the explosion of cylindrical tanks caused by the BLEVE effect. The initial velocity and aerodynamic characteristics define the shape of the trajectory and range of the fragment. The estimation of the fragment kinematic parameters is of crucial importance for an adequate 
setting of fragmentation barriers. The fragmentation barriers have a role of protecting process installations from the fragmentation effect [6-7]. The basic characteristics of a fragment are defined by the number, shape, speed, and trajectory [8].

Basic research in terms of defining the mass of generated fragments of cylindrical tanks used for the storage of liquefied petroleum gas (LPG) was first done by Baker et al. [9]. Mass distribution from the previous study served as a basis for several recent pieces of research in the field of fragmentation of tanks [10-11]. Fragmentation analysis serves to define preventive measures to protect process installations from a potential fragment impact [12]. The fragmentation analysis in the presented study is limited to the identification of kinematic parameters. The most important kinematic parameter in the fragmentation of a tank is the initial velocity. According to the literature, the initial velocity estimation is carried out on the basis of the energy released in the tank explosion, in accordance with the models presented in [13-14]. The kinetic energy corresponding to the initial velocity of a fragment is estimated on the basis of the share of explosion energy [15]. The factor $\alpha$ of the explosion energy share transferred to the fragment is between $20 \%$ and $50 \%$; this factor was defined by Hauptmanns et al. [10]. The estimation of this factor in the range of $5 \%$ to $20 \%$ was proposed by Tugnoli et al. [16]. Accordingly, the aforementioned literature sources do not give corresponding values for the factor $\alpha$ and the initial velocity. In this context, the objective of our paper is to develop an alternative procedure for assessing the initial velocities integrated into a dynamic fragmentation model. The dynamic analysis of a fragment is the basis for identifying the fragmentation mechanism which is interpreted in the literature through a simplified mathematical model [17]. This fragmentation model, as generally accepted in the literature, was proposed by Mannan [18]. It has been experimentally proven that the initial velocity increases with the fragment mass [19]. The indicated dependence of mass on the initial velocity of the fragment should be consistent with the results of the fragmentation model presented here. The polar and azimuth angles corresponding to the initial velocity have a uniform distribution according to Mébarki et al. [15]. The use of this assumption is justified in the fragmentation model. In the continuation of the paper, an original fragmentation model with a focus on the initial velocity, the shape of the trajectory, and the range of fragments will be presented. The significance and contribution of the model developed here should be reflected in a much better estimate of a fragmentation risk than that presented in the literary source [20] and verified by accidental data $[16,21]$.

\section{Stress analysis of the tank}

Horizontal cylindrical tanks for the storage of LPG are reliable technical systems designed according to EN 13445-3 [22]. The designed exploitation characteristics and the achieved quality of production are checked by testing the tank according to EN 13445-5 [23]. The two-axis stress state of the tank indicates the longitudinal and the radial deformation of the shell. The analysis of stress state of the tank is an integral part of design activities in terms of fulfilling the exploitation requirements. A typical shape of a horizontal cylindrical tank discussed in the continuation of the paper is given in Fig. 1. The construction of the tank consists of supports (item 1), cylinder segments (items 2-5), elliptical end caps (item 6) and lifting lugs (item 7). The tank is supplied with a filling and a discharging system (FDS), a measure and a control system (MCS), an inspection hatch (IH), and a safety valve (SV). The mass of empty tank is $12,300 \mathrm{~kg}$, and it provides storage for up to 50,000 l of TNG.

\subsection{Theoretical analysis of the tank}

The exact analytical expressions for the stresses of the considered axially symmetric cylindrical tank with elliptical end caps according to [24] are: 


$$
\begin{aligned}
& \sigma_{x}=\left[1+\frac{3}{2} \frac{1}{\sqrt{3\left(1-v^{2}\right)}}\left(\frac{D}{2 h}\right)^{2} e^{-\lambda x} \sin (\lambda x)\right] \cdot\left(\frac{D p}{4 \delta}\right)=46.5 p \\
& \sigma_{\theta}=\left[1+\frac{1}{4}\left(\frac{3 v \sin (\lambda x)}{\left.\left.\sqrt{3\left(1-v^{2}\right)}-\cos (\lambda x)\right)\left(\frac{D}{2 h}\right)^{2} e^{-\lambda x}\right] \cdot\left(\frac{D p}{2 \delta}\right)=92.5 p,}\right.\right. \\
& \sigma_{c r}=\sqrt{\sigma_{x}^{2}+\sigma_{\theta}^{2}-\sigma_{x} \sigma_{\theta}+\frac{3}{2}\left(\sigma_{x}-\sigma_{\theta}\right)^{2}}=98 \cdot p_{c r} .
\end{aligned}
$$

Where the coefficient $\lambda$ is determined as:

$$
\lambda=\sqrt[4]{\frac{12\left(1-v^{2}\right)}{D^{2} \delta^{2}}}
$$

Horizontal cylindrical tanks have three critical cross-sections (Figure 1). A critical cross-section A-A is characteristic of tanks with torispherical end caps, while elliptical end caps influence the tank fracture in the B-B cross-section (Figure 1a). Fractures along the cross-section $\mathrm{C}-\mathrm{C}$ occur exclusively in the tanks with spherical end caps (Figure 1b). The wall thickness of the tank is constant, $\delta=14 \mathrm{~mm}$ (Figure 1c). This condition is of great importance in the fragmentation model used for the assessment of initial velocity.

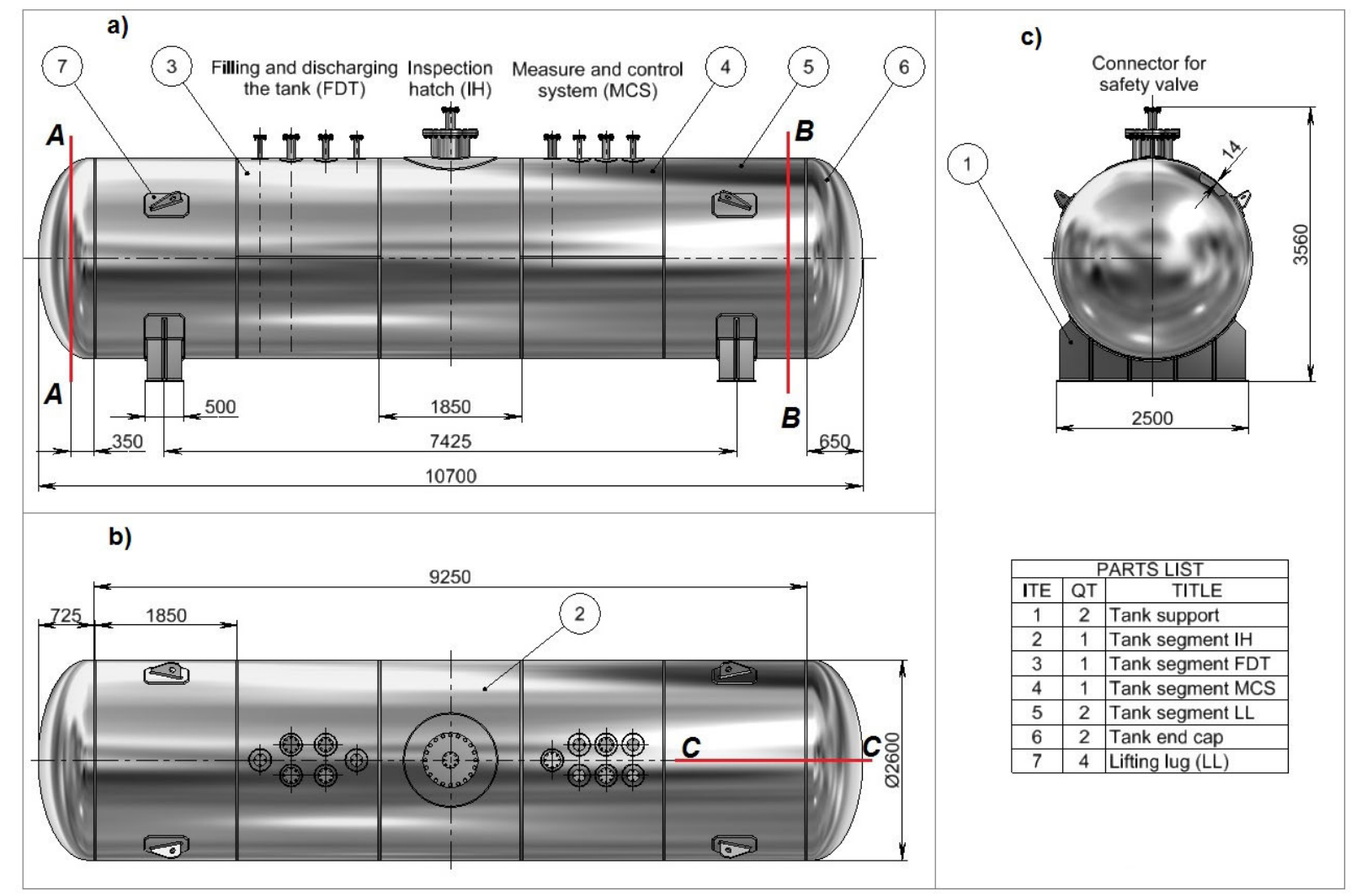

Fig. 1 A horizontal cylindrical tank with elliptical end caps according to DIN 28013

The critical zone of the tank in Fig. 1 corresponds to the transition of the cylinder to the elliptical end cap (section B-B). The relevant stress for sizing the tank under stress is given by expression (3). The maximum permissible tank stress $\mathrm{f}$ is determined based on the material type (S355J2G3) according to EN 13445-3 [22]: 


$$
f=\min \left\{\begin{array}{l}
R_{e H} / 1.5 \\
R_{m} / 2.4
\end{array}\right\}=\min \left\{\begin{array}{l}
355 / 1.5 \\
470 / 2.4
\end{array}\right\}=195.83 \mathrm{MPa}
$$

The maximum permissible operating pressure $p_{\text {theory }}$ is determined from (3) according to (5), and it amounts to $2 \mathrm{MPa}$. This pressure, according to EN 13445-3, is expressed as $p_{E N 13445-3}=2 \cdot f \cdot z \cdot e_{a} / D_{m}=2.12 \mathrm{MPa}$, where the mean diameter of the tank is $D_{m}=\left(D-e_{a}\right)=$ $2586 \mathrm{~mm}$, the thickness of the sheet $e_{a}=\delta=14 \mathrm{~mm}$, and the coefficient of welded seam $z=1$ (complete control of welded seams). The maximum operating pressure calculated according to EN 13445-3 is practically the membrane stress in the radial direction corrected by factor $z$. The operating pressure of the TNG storage tank ranges from 1.64 to $1.69 \mathrm{MPa}$ (an average of $1.67 \mathrm{MPa}$ ), while the test pressure $p_{\text {test }}$ is $50 \%$ higher than the operating pressure and amounts to $2.5 \mathrm{MPa}$. The maximum equivalent stress during the test is $245 \mathrm{MPa}$ and is $30 \%$ lower than $R_{e H}$, indicating the inability of plastic deformation to occur during the test. The distribution of impact pressure in the pressure vessels under the influence of the impact excitation of interest (sloshing), obtained by measurements, is shown in the paper [25]. The above data clearly indicate that the tank of the given geometry is correctly designed for the function of LPG storage.

\subsection{Physical explosion and the BLEVE effect}

The way in which pressure within a tank is created plays an important role in its fragmentation. There are two characteristic cases of the tank loading: the first, when the internal pressure is static (quasi-static) and the second, when it is dynamic. The type of stress has a direct effect on the number of generated fragments, but not on the kinematic sizes. The quasi-static pressure of fragmentation occurs in a gradual increase in the fluid pressure until the tank fractures. The destruction of the tank at the quasi-static pressure is accompanied by a less pronounced fragmentation effect, which implies a lower number of generated fragments. The quasi-static pressure causes a tank fracture in the zone of stress concentration due to potential errors (cracks, faults in welded seams, corrosion, etc.). The destruction of the tank due to the dynamic pressure is characterised by an explosion and the appearance of a spherical longitudinal wave of high frontal velocity (of up to several $\mathrm{km} / \mathrm{s}$ ). The velocity of the longitudinal wave spreading through the steel tank amounts only to $c=(E / \rho)^{0.5}=163.6 \mathrm{~m} / \mathrm{s}$ and it is usually lower than the velocity of explosion wave by a whole order of magnitude [26]. This fact leads to the conclusion that the dynamic pressure affects mostly the inner zones of the tank, while for its transfer to the surface layers, the time $t=\delta / c=8.55 \cdot 10^{-5} \mathrm{~s}$ is required. During this time, the explosion wave can cross a length of $85.5 \mathrm{~mm}$, assuming its velocity is $1 \mathrm{~km} / \mathrm{s}$. It is quite obvious that in these conditions, the radial deformation of the tank would be $\varepsilon_{\theta}=\Delta D / D=6.3 \%$, which is not possible to achieve even theoretically since the destruction of the tank occurs at a significantly lower value of deformation, i.e. at

$\varepsilon_{\theta, \text { failure }}=[1-D /(2 \delta)] \cdot\left(R_{m} / E\right)=0.025 \%$. These numerical values should explain the reason for the formation of a larger number of fragments during fragmentation. They should also show that the number of fragments increases with the velocity of the detonation frontal wave. The quasi-static pressure has enough time to find the source of stress concentration on the surface layer, where the crack and the fracture of the tank are spreading from. The dynamic pressure manifested through a shock detonation wave caused by an explosion does not have enough time to find the most critical source of stress concentration most commonly found on the outside of the tank, but it locates a local one from the inside of the cylinder (usually a spot with strong corrosion), where the formation of a crack line starts. Stress lines, before they are localized in one critical zone on the surface layer, are interrupted due to plastic deformation, i.e. fracture of the tank. 
Thus, the relatively slow progression of the stress through the thickness of the shell and the high velocity of the detonation wave are the main causes of the generation of a large number of fragments. Naturally, as these contrasts are sharper, the number of primary fragments will be larger.

\section{Fragmentation model}

Identification of kinematic parameters that are relevant for the assessment of fragmentation hazards is carried out by the dynamic analysis of fragments. The basic kinematic parameters include the initial velocity $\left(v_{0}\right)$, the polar angle $(\varphi)$, and the aerodynamic characteristics of the fragments $\left(C_{D}\right.$ and $\left.C_{L}\right)$. The fragmentation model is based on the exact equation of motion of a fragment in a resistive environment, with the initial velocity $v_{0}$ and the launch angle $\varphi_{0}$.

\subsection{The dynamics of a fragment}

The flight of all fragments is realized under the influence of inertial, gravitational and thrust forces, as well as the forces of air resistance. The dynamics of the flight of a fragment which has the mass $m_{f r}$ and the velocity $v_{f r}$ is described by an ordinary differential equation [27]:

$$
m_{f r} \cdot \frac{\mathrm{d} \vec{v}_{f r}}{\mathrm{~d} t}=\vec{F}_{D}+\vec{F}_{L}+\vec{G}
$$

The force of the air resistance during the fragment flight is defined by the expression:

$$
\vec{F}_{D}=-\left(\frac{1}{2} \rho_{v} C_{D} A_{D} v_{f r}\right) \cdot \vec{v}_{f r}
$$

The thrust force during the fragment flight is:

$$
\vec{F}_{L}=-\left(\frac{1}{2} \rho_{v} C_{L} A_{L} v_{f r}\right) \cdot \vec{v}_{f r}
$$

The trajectories are analysed in the vertical plane $O x z$ of the local coordinate system $O x y z$ (Figure 2). The projections of the vector equation of motion (5) on the axes of the local coordinate system are given by expressions (9) - (11).

Direction $x$ :

$$
m_{f r} \cdot\left(\frac{\mathrm{d} v_{f r}}{\mathrm{~d} t}\right)_{x}+\left(\frac{1}{2} \rho_{\nu} C_{D} A_{D} v_{f r}\right) \cdot\left(v_{f r} \cos \varphi\right)+
$$

$$
+\left(\frac{1}{2} \rho_{v} C_{L} A_{L} v_{f r}\right) \cdot\left(v_{f r} \sin \varphi\right)=0
$$

Direction $y: \quad m_{f r} \cdot\left(\frac{\mathrm{d} v_{f r}}{\mathrm{~d} t}\right)_{y}=0$

Direction $z$ :

$$
m_{f r} \cdot\left(\frac{\mathrm{d} v_{f r}}{\mathrm{~d} t}\right)_{z}+\left(\frac{1}{2} \rho_{v} C_{D} A_{D} v_{f r}\right) \cdot\left(v_{f r} \sin \varphi\right)-
$$

$$
-\left(\frac{1}{2} \rho_{v} C_{L} A_{L} v_{f r}\right) \cdot\left(v_{f r} \cos \varphi\right)+m_{f r} g=0
$$


It can be shown easily that the final form of the equations of fragment motion (9) and (11) are equivalent to equations (12) and (13) which represent the balance equations of the inertial acceleration $a_{f r}$ with the resistive and the thrust acceleration $a_{d r a g}$ and $a_{\text {liff }}$, respectively.

$$
\begin{aligned}
& a_{x, f r}=a_{\text {drag }} \cdot(-\cos \varphi)+a_{\text {lift }} \cdot(-\sin \varphi) \\
& a_{z, f r}=a_{\text {drag }} \cdot(-\sin \varphi)+a_{\text {lift }} \cdot(\cos \varphi)-g
\end{aligned}
$$

The previous system of equations gives a possibility of defining the polar angle $\varphi$ during the flight of a fragment, which is particularly important at the starting and the end point. The parameter $\varphi$ represents the angle that the velocity vector forms with the $x$ axis. The ascending part of the trajectory is characterized by $\varphi>0$, and the descending part by $\varphi<0$. The direction of velocity $\varphi$ is a function of the intensity of the inertial and the gravitational acceleration, as well as of the relation of the aerodynamic resistance to the thrust of the fragment; this dependence is expressed as:

$$
\varphi=\operatorname{arctg}\left(\frac{v_{z, f r}}{v_{x, f r}}\right)=\operatorname{arctg} \frac{\left(\frac{k_{L}}{k_{D}}\right) \cdot\left(-a_{x}\right)+\left(-a_{z}\right)-g}{\left[\left(-a_{x}\right)+\left(\frac{k_{L}}{k_{D}}\right) \cdot a_{z}+g\right]}
$$

The initial polar angle $\varphi_{0}$ represents the launch angle of a fragment, which is uniquely defined by the knowledge of its initial acceleration and shape. This proves that the direction of initial velocity of the fragment is not the stochastic size of uniform distribution as assumed in [15], but the deterministic parameter of the expressed dissipation followed by the epistemic uncertainty. The uncertainty in defining the launch angle of the fragment results from the dissipation of inputs $\left(a_{0}, k_{L}\right.$, and $\left.k_{D}\right)$ and is eliminated by representing the range in the form of a probability density function (Figures 3 and 4 ).

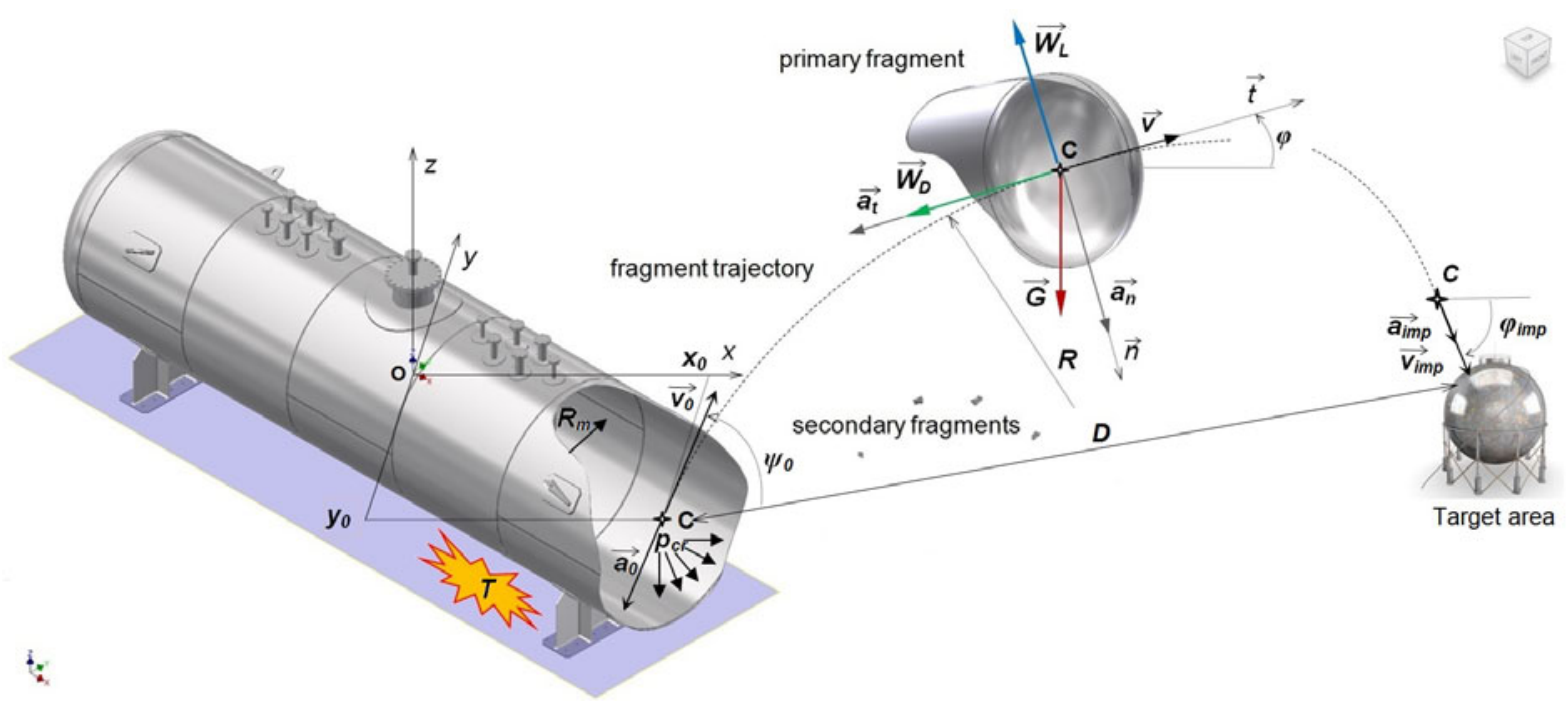

Fig. 2 Fragmentation of a horizontal cylindrical tank

The launch angle of a fragment is essential for the calculation of the range and assessment of fragmentation risk, while the knowledge of velocity direction in the final phase of the flight is necessary for the analysis of mechanical damage to adjacent installations, as well as for the assessment of the risk due to the domino effect. Various directions of impacts with the same kinetic energy of the fragment are completely different in terms of mechanical 
damage to the process equipment. The velocity vector components $v_{x, f r}$ and $v_{z, f r}$ are obtained from the final equations of motion of the fragments given by (15) and (16):

$$
\begin{aligned}
& v_{x, f r}=\sqrt{\frac{2 m_{f r} \cdot\left(-a_{x}\right)}{\rho_{\text {air }} \cdot\left[C_{D} A_{D}+C_{L} A_{L} \cdot \operatorname{tg} \varphi\right]}} \cdot \frac{1}{\sqrt[4]{1+\operatorname{tg}^{2} \varphi}} \\
& v_{z, f r}=\sqrt{\frac{2 m_{f r} \cdot\left(-a_{x}\right)}{\rho_{\text {air }} \cdot\left[C_{D} A_{D}+C_{L} A_{L} \cdot \operatorname{tg} \varphi\right]}} \cdot \frac{\operatorname{tg} \varphi}{\sqrt[4]{1+\operatorname{tg}^{2} \varphi}}=v_{x, f r} \cdot \operatorname{tg} \varphi
\end{aligned}
$$

The intensity of velocity of a fragment is determined by:

$$
v_{f r}=\sqrt{v_{x, f r}^{2}+v_{z, f r}^{2}}
$$

The initial velocity is a deterministic parameter in the initial acceleration, the shape, and the mass of the fragment. Dissipation of this parameter is considered integrated with the launch angle through a corresponding statistical distribution of the range. The initial directions of the velocity and acceleration vectors are not collinear since the beginning of the flight of all fragments is characterized by the appearance of thrust $\left(C_{L} \neq 0\right)$ and air resistance $\left(C_{D} \neq 0\right)$. The offensive velocity angle (14) is a function of the acceleration components $\left(-a_{x}\right)$ and $\left(-a_{z}\right)$, as well as of the ratio of thrust to air resistance $k_{L} / k_{D}$. The $k_{L} / k_{D}$ factor physically represents the ratio of the thrust force to the force of air resistance during the fragment flight and its value influences the deviation of the direction of the initial velocity and acceleration vectors. The initial conditions of the fragment defined in relation to the local coordinate system $O x y z$ immediately after the tank explosion $\left(t=t_{o}=0\right)$ are given by the following expressions:

$$
\begin{aligned}
& \left(x_{f r}\right)_{0}=x_{0} \wedge\left(z_{f r}\right)_{0}=z_{0} \\
& \left(v_{x, f r}\right)_{0}=v_{x o} \wedge\left(v_{z, f r}\right)_{0}=v_{z o}
\end{aligned}
$$

The trajectory of the fragments is obtained by solving the finite equations of motion (9) and (11) using the initial conditions (18) and (19). Considering the impossibility of an analytic solution due to the interconnection between the equations, a numerical procedure based on the Taylor's series method is applied. This method is characterized by simplicity in programming, quick determination of error and exact range values. The numerical solution algorithm (9) - (11) that includes the following steps is given below:

Step 1: Initialisation of the starting parameters $-m_{f r}, R_{m}, \rho, \delta, C_{D}, C_{L}, A_{D}, A_{L}, \varphi_{0}$.

Step 2: Discretisation of time $-\Delta t_{n}$.

Step 3: Definition of the initial conditions $-x_{0}, z_{0}, v_{x 0}, v_{z 0}$.

Step 4: Calculation of the fragment acceleration $-a_{f r}$.

$$
\text { For } i=0 \rightarrow \text { see }(20)
$$

Step 5: Calculation of the speed of a fragment $-v_{f r}$.

$$
v_{f r}\left(t_{i+1}\right)=\sqrt{\left[a_{x, f r}\left(t_{i}\right) \cdot(\Delta t)\right]^{2}+\left[a_{y, f r}\left(t_{i}\right) \cdot(\Delta t)\right]^{2}}
$$


Step 6: Calculation of the trajectory coordinates $-\left(x_{i+1}, z_{i+1}\right)$

$$
\begin{aligned}
& x\left(t_{i+1}\right) \approx x\left(t_{i}\right)+v_{x}\left(t_{i}\right) \cdot \Delta t+\frac{1}{2} a_{x}\left(t_{i}\right) \cdot(\Delta t)^{2} \\
& z\left(t_{i+1}\right) \approx z\left(t_{i}\right)+v_{z}\left(t_{i}\right) \cdot \Delta t+\frac{1}{2} a_{z}\left(t_{i}\right) \cdot(\Delta t)^{2}
\end{aligned}
$$

Loop 1: If $z\left(t_{1}\right)>0 \rightarrow$ continue the procedure,

Loop n: If $z\left(t_{i+1}\right)<0 \rightarrow$ stop

Step 7: Printing for $i=n-1$.

$$
x\left(t_{n}\right) ; z\left(t_{n}\right) ; v_{f r}\left(t_{n}\right) ; a_{f r}\left(t_{n}\right)
$$

Step 8: Reduce the discretisation time interval $\Delta t_{N}$

Step 9: Repeat the procedure defined by:

Steps 4, 5, and 6

$$
\text { Loops from } i=1 \text { to } i=N(n<N)
$$

Step 10: Printing for $i=N-1$.

$$
x\left(t_{N}\right) ; z\left(t_{N}\right) ; v_{f r}\left(t_{N}\right) ; a_{f r}\left(t_{N}\right)
$$

Step 11: Calculation of the exact value of the range $-R_{\text {egz }}$

$$
R_{e g z}=\frac{z\left(t_{N}\right)-z\left(t_{n}\right)}{\Delta t_{n}-\Delta t_{N}} \Delta t_{n}
$$

Step 12: Check the convergence of the range:

$$
R_{e g z}=\lim _{\Delta t_{n}=0} x\left(t_{n}\right)
$$

Step 13: Generate trajectories with a set of coordinates:

$$
\left(x_{N}, z_{N}\right)
$$

Stop!

\subsection{Initial acceleration of a fragment}

Initial acceleration is the basic kinematic parameter of a fragment; it is determined based on the physical resistance of the tank material. Initial acceleration is a parameter required for defining other kinematic parameters, such as velocity and trajectory. However, the literature in the field of tank fragmentation uses the explosive energy of the tank instead of this kinematic parameter. In the literature, the initial velocity is determined by the kinetic energy of a fragment estimated on the basis of the explosive energy of the tank [8, 15-16]. However, the basic problem of such a procedure is how to estimate the share of the kinetic energy of each fragment in the total amount of energy generated by the explosion. All procedures proposed in the literature are either not clearly explained or are based on a rather approximate approach.

In the introductory part of this paper it was emphasized that the share of kinetic energies of fragments ranges from $20 \%$ to $50 \%$ of the explosive energy of the tank, which clearly indicates considerable dissipation of the initial velocity [8]. The estimate of the share of the kinetic energy of fragments based on the maximum entropy principle was suggested by Mébarki et al. [15]. The results of that approach show that the coefficient $\alpha$ does not exceed the value of 0.24 , and that it has a very low probability of occurrence. If these results are compared with the distribution proposed by Hauptmanns [11], the qualitative and the quantitative disagreement between the two can clearly be established. 
In his study, Hauptmanns [11] proposed the prediction of the share of kinetic energy of fragments by their masses, as well as the coefficient $\alpha$ with a triangular distribution of the expected value of 0.3 . In the same research, it was found that the greater mass of a fragment corresponds to a larger share of explosive energy. These facts show that in most cases there are fragments with a share of kinetic energy of $\alpha=0.24$ in the explosive energy of the tank.

The motion of fragments is realized under the influence of the impulse load force $F$ created by the minimal critical pressure $p_{c r}$ and distributed over the surface of the generated fragment $A$; the average value of the impulse load force $F$ is expressed as $p_{c r} \cdot A$. This force acting on a fragment of the mass $m_{f r}$ generates the acceleration $a_{o}$ expressed as:

$$
a_{o}=\frac{F}{m}=\frac{p_{c r}}{\rho \delta}=\frac{R_{m}}{104 \rho \delta}=\text { const }
$$

The preceding expression shows that the initial acceleration is a constant quantity for a particular type of tanks and that each fragment in the tank explosion is characterised by the same velocity change in a unit of time. Inertial forces of fragments differ, taking into consideration their different masses. The advantage of introducing initial acceleration in the fragmentation analysis is reflected in the use of the minimum critical pressure $p_{c r}$ which is characteristic of the tank type and not of the generated explosive energy. Namely, the explosive pressure $p_{e}$ inside the tank depends on the explosive energy and it acts on the tank only until it reaches the value of $p_{c r}$ when fragmentation occurs. Upon the occurrence of cracks, the explosive pressure suddenly decreases; thus, the fragmentation always fulfils the condition $p_{c r}<<p_{e}$. Therefore, the minimum critical pressure $p_{c r}$ is of major importance for the effect of tank fragmentation; it can be accurately estimated on the basis of the stress state of the tank itself, while the ratio of the pressure $p_{c r}$ to the pressure $p_{e}$ is important for defining the number of generated fragments.

\section{Shapes of trajectories and the height of the target}

Dynamic analysis of the flight of fragments indicates three characteristic shapes of trajectories: i) curved, ii) peaked, and iii) gradually rising curve with a sharp fall after the peak. The curved shape occurs when there is almost no fragment thrust, while the peaked shape occurs with aerodynamic shapes of fragments. The gradually rising curve with a sharp fall after the peak is a combination of the previous two variants and it is the most probable trajectory in the flight of fragments. This shape is characterised by the average values of air resistance and thrust of the fragment. Trajectory shapes for different aerodynamic properties of fragments of the same mass are shown in Figures 3 and 4. Fragmentation characteristics are classified into fixed and variable parameters. The fixed parameters $\left(m_{f r}, \psi_{0}, \delta, f\right)$ represent constants for all trajectory cases (Case 1-20). The variable parameters are different for each case of the trajectory of a fragment, and include the coefficients $k_{L}$ and $k_{D}$. The estimation of coefficients $k_{L}$ and $k_{D}$ is done on the basis of the minimum and maximum values of $C_{D}$ and $C_{L}$ whose recommended values are given in [15]. The combination of different values of $k_{L}$ and $k_{D}$ gives the potential trajectories of a fragment for given fixed parameters (Figures 3 and 4). The ranges of these trajectories are used as inputs for the Monte Carlo simulation. The aim of the Monte Carlo simulation is to identify the distribution of the range for a fragment with characteristics of the fixed parameters (Figures $3 \mathrm{c}$ and $4 \mathrm{c}$ ). This enables a probabilistic estimate of the range of the reserve for a given mass and direction of the fragment launch.

The target height represents an obstacle to the flight of a fragment and has a strong effect on the fragmentation risk. Targets of a lesser height and lower base face a lower risk. In this regard, a fragment of a mass of $200 \mathrm{~kg}$, launched at an angle of $5^{\circ}$ is considered in different aerodynamic conditions (Table 1). Fragmentation parameters, i.e. the achieved height $h(\mathrm{~m})$, 
the flight time $t(\mathrm{~s})$, and the velocity of the fragment $v(\mathrm{~m} / \mathrm{s})$ are analysed at distances of $50 \mathrm{~m}$, $100 \mathrm{~m}, 150 \mathrm{~m}$, and $200 \mathrm{~m}$ for a maximum target height of $15 \mathrm{~m}$. Aerodynamic shapes of fragments can guarantee that at a distance of $50 \mathrm{~m}$, a fragment will fly over a $15 \mathrm{~m}$ high target. Fragments with bad aerodynamic properties can endanger a $15 \mathrm{~m}$ high target even at distances larger than $150 \mathrm{~m}$.

a)

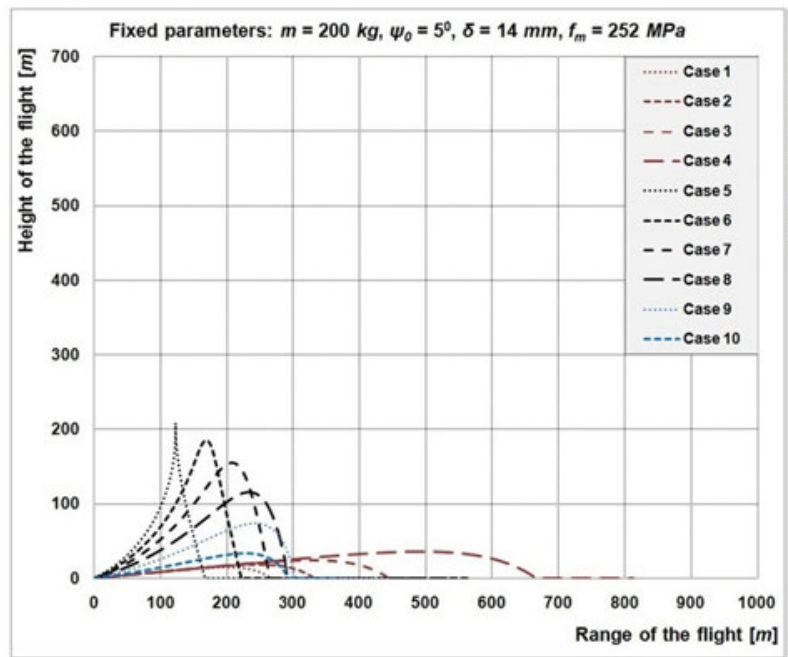

b)

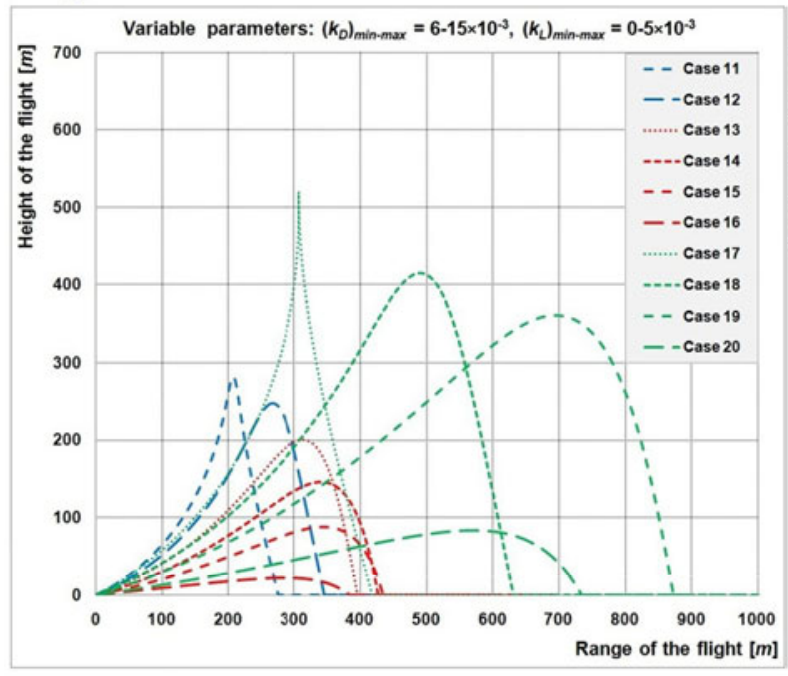

c)

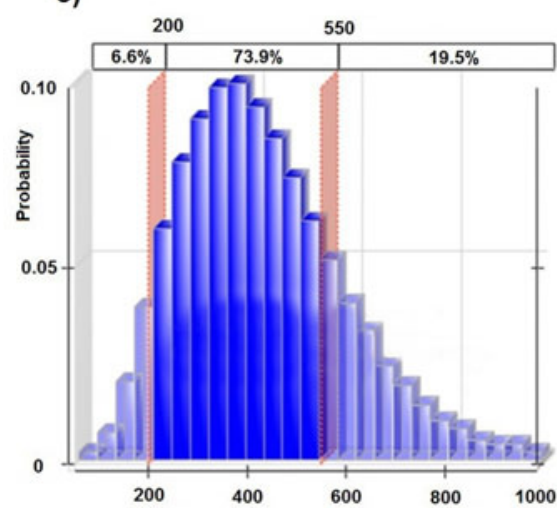

Fig. 3 Trajectory shapes (a and b), and distribution of the range for a fragment with characteristics of the fixed parameters $m_{f r}=200 \mathrm{~kg}, \psi_{0}=5^{\circ}(\mathrm{c})$ a)

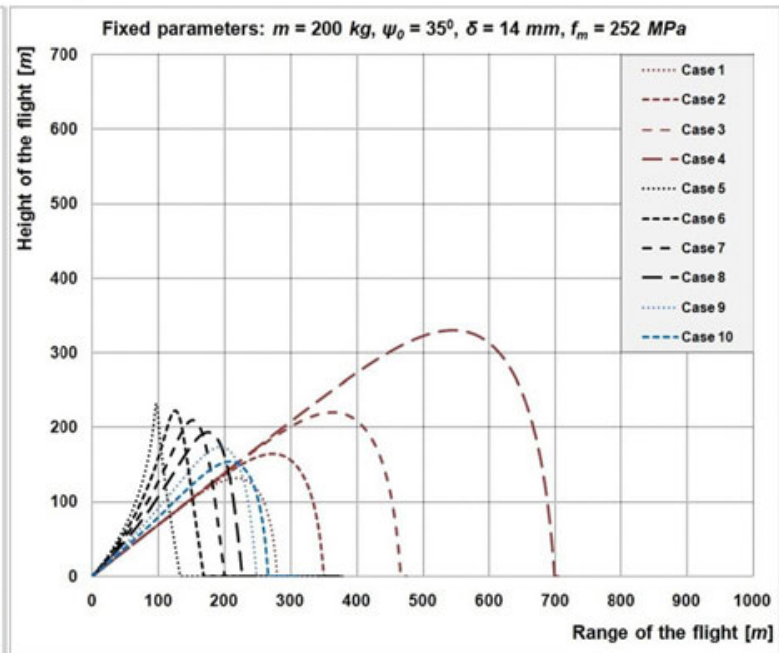

b)

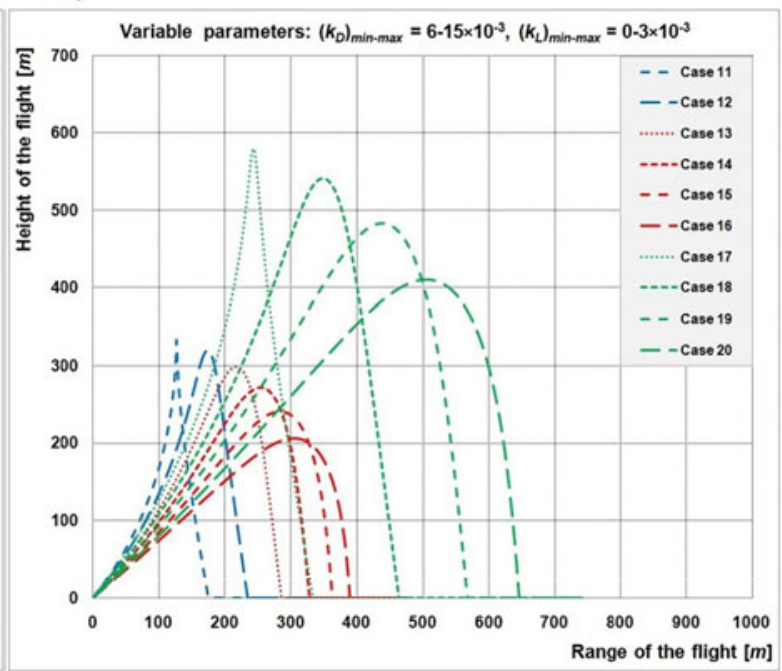

c)
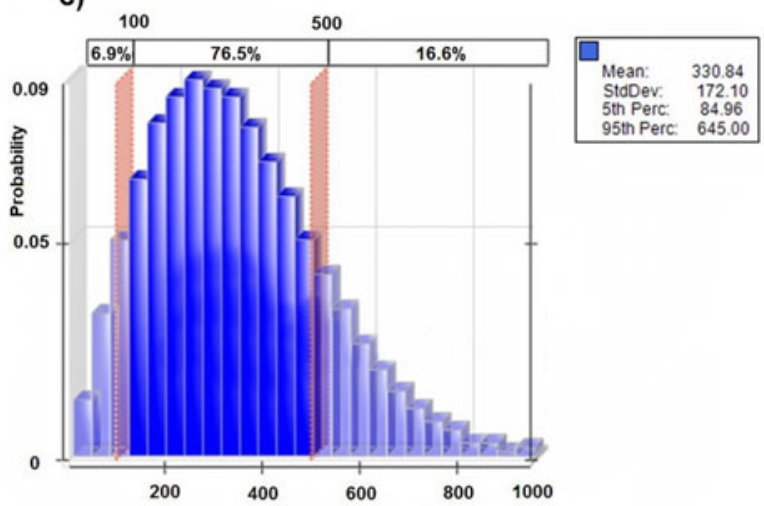

Fig. 4 Trajectory shapes (a and b) and distribution of the range for a fragment with characteristics of the fixed parameters $m_{f r}=200 \mathrm{~kg}, \psi_{0}=35^{\circ}$ (c) 
Expected values of the thrust acceleration coefficient $k_{L}$ for a fragment of a mass of $200 \mathrm{~kg}$ must be greater than $0.0021 \mathrm{~m}^{-1}$. The average impact velocity of the fragment of a mass of $200 \mathrm{~kg}$ at a distance of $200 \mathrm{~m}$ is $97 \mathrm{~m} / \mathrm{s}$ and it is 3 times higher than the average impact velocity at the base of the target at a distance of $396 \mathrm{~m}$. Smaller fragments have better aerodynamic properties since they have a shell shape in most of the cases.

Table 1 Parameters for estimating fragmentation hazards with the height of the target

\begin{tabular}{|c|c|c|c|c|c|c|c|c|c|}
\hline \multirow{2}{*}{ Distance } & \multicolumn{4}{|c|}{ Mass of the fragment: $200 \mathrm{~kg}$} & \multirow{2}{*}{ Distance } & \multicolumn{4}{|c|}{ Mass of the fragment: $200 \mathrm{~kg}$} \\
\hline & $50 \mathrm{~m}$ & $100 \mathrm{~m}$ & $150 \mathrm{~m}$ & $200 \mathrm{~m}$ & & $50 \mathrm{~m}$ & $100 \mathrm{~m}$ & $150 \mathrm{~m}$ & $200 \mathrm{~m}$ \\
\hline Case 1 & 4.330 & 8.580 & 12.400 & 14.300 & Case 4 & 4.390 & 8.670 & 12.980 & 17.250 \\
\hline$k_{L}=0.0000$ & 0.062 & 0.195 & 0.47 & 1.077 & $k_{L}=0.0000$ & 0.031 & 0.072 & 0.128 & 0.204 \\
\hline$k_{D}=0.0150 v[\mathrm{~m} / \mathrm{s}]$ & 564 & 264 & 124 & 59 & $k_{D}=0.0060$ & 1,404 & 1,040 & 769 & 568 \\
\hline Case 6 & 25.550 & 69.600 & 153.27 & 83.740 & Case 10 & 13.140 & 30.210 & 51.470 & 77.060 \\
\hline$k_{L}=0.0041$ & 0.075 & 0.303 & 1.602 & 9.885 & $k_{L}=0.0014$ & 0.048 & 0.133 & 0.287 & 0.574 \\
\hline$k_{D}=0.0150 v[\mathrm{~m} / \mathrm{s}]$ & 421 & 120 & 14 & 6 & $k_{D}=0.0105$ & 796 & 444 & 243 & 129 \\
\hline Case 11 & 9.340 & 20.610 & 34.130 & 49.540 & Case 13 & 25.300 & 58.040 & 100.72 & 157.77 \\
\hline$k_{L}=0.0008 \quad t[\mathrm{~s}]$ & 0.047 & 0.127 & 0.268 & 0.515 & $k_{L}=0.0020$ & 0.036 & 0.090 & 0.177 & 0.332 \\
\hline$k_{D}=0.0105 v[\mathrm{~m} / \mathrm{s}]$ & 823 & 474 & 272 & 155 & $k_{D}=0.0060$ & 1,150 & 741 & 445 & 237 \\
\hline Case 15 & 13.460 & 29.650 & 47.760 & 68.550 & Case 17 & 18.260 & 43.460 & 76.620 & 119.73 \\
\hline$k_{L}=0.0008$ & 0.028 & 0.066 & 0.115 & 0.181 & $k_{L}=0.0021$ & 0.048 & 0.136 & 0.305 & 0.659 \\
\hline$k_{D}=0.0050 v[\mathrm{~m} / \mathrm{s}]$ & 1,545 & 1,165 & 880 & 658 & $k_{D}=0.0100$ & 787 & 422 & 212 & 97 \\
\hline
\end{tabular}

The paper deals with the cases when a fragment of a mass of $200 \mathrm{~kg}$ is launched at angles of $5^{\circ}$ and $35^{\circ}$. The estimation of the shape and mass of the fragment was carried out on the basis of accidents data [16,21]. Low-thrust fragments are characterized by a low height of the flight (Cases 1-4). An increase in the thrust of a fragment results in an increase in the range, but only up to the average values of $k_{L}$. Excessive thrust of the fragment is accompanied by a dramatic increase in the height of the flight and a decrease in the range (Cases 5 and 17). The fluctuation effect of air resistance and thrust requires a statistical interpretation of the fragment range; therefore, the Monte Carlo simulation was applied for this purpose.

\section{Results and discussion}

The relevant inputs of the fragmentation risk model include the kinematic parameters of the generated fragments. The range of a fragment is the most important kinematic parameter, and its representation in the form of statistical distribution allows a probabilistic assessment of the impact of the fragment in the targeted areas. A potential vulnerability of targets due to the fragmentation effect depends on the kinetic energy that increases with the square of velocity of the fragment. Reliability of the assessment of fragmentation hazards depends on the adequately estimated velocity during the flight of fragments. Besides the impact velocity $v_{i m p}$, the angle of fragment impact on the target $\varphi_{\text {imp }}$ and the fragment acceleration $a_{i m p}$ play important roles in the fragmentation hazard assessment. The acceleration effect should only be considered when the angle of impact is $\varphi_{\text {imp }}< \pm 60^{\circ}$. The kinematic parameters that are relevant for estimating fragmentation hazards are given in Table 2. The fluctuation effect of air resistance and thrust during the flight of a fragment requires a probabilistic approach to the estimation of the range. The results of Monte Carlo simulations show that the range of a fragment of a mass of $200 \mathrm{~kg}$, launched at an angle of $5^{\circ}$, can be described by the gamma function with the parameters 6.28 and 66.66. The range of a fragment of the same mass that is launched at an angle of $35^{\circ}$ is adequately described by the Rayleigh function with the 
parameter 264.43. In the first case, the expected value of the range is 416.25 with a probability of $10 \%$; in the second case, the range is $330.84 \mathrm{~m}$ with a probability of $9 \%$. Estimation of the range of a fragment makes sense only if the value is followed by the corresponding probability; otherwise, the range is accompanied by epistemic uncertainty. Thus, the fragment of a mass of $200 \mathrm{~kg}$ launched at an angle of $5^{\circ}$ has a probability of only $6.6 \%$ to have a range of up to $200 \mathrm{~m}$ and of $19.5 \%$ to have a range over $550 \mathrm{~m}$.

Thus, for the fragment analysed above, the range is between $200 \mathrm{~m}$ and $550 \mathrm{~m}$ with a probability of $73.9 \%$. A fragment of the same mass launched at an angle of $35^{\circ}$ has a range between $100 \mathrm{~m}$ and $500 \mathrm{~m}$ with a probability of $76.5 \%$. The aforementioned data show that the average range values decrease with an increasing angle of launch of a fragment. The basic kinematic parameters for the typical cases of the fragment flight include the initial velocity $v_{0}$, the range $R$, the impact velocity $v_{i m p}$, the direction of impact $\varphi_{i m p}$, and the acceleration $a_{i m p}$ (Table 2). Cases 1 and 2 are characterised by the absence of thrust, assuming the weak aerodynamicity of the fragment. Air resistance is always present, therefore $k_{D}>0$ in all the cases. Inputs of the fragmentation model integrated with the Monte Carlo simulation provide the ability to estimate the most reliable trajectory of a fragment for given fixed parameters. The most likely form of the trajectory for a fragment of a mass of $200 \mathrm{~kg}$ that was launched at an angle of $5^{\circ}$ corresponds to Case $14\left(k_{L}=1.4 \cdot 10^{-3} \mathrm{~m}^{-1}\right.$ and $\left.k_{D}=1.05 \cdot 10^{-2} \mathrm{~m}^{-1}\right)$. The launch speed in this case is $1,442 \mathrm{~m} / \mathrm{s}$, which allows the fragment to reach a range of $427 \mathrm{~m}$ and an impact velocity of $29.5 \mathrm{~m} / \mathrm{s}$. The fragment hits the target at a very steep angle $\left(-78.2^{\circ}\right)$ at an acceleration of $1 \mathrm{~m} / \mathrm{s}^{2}$. Acceleration can be ignored in the given case, according to the literature sources [15].

Table 2 Kinematic fragmentation parameters for $m_{f r}=200 \mathrm{~kg}$ and $\psi_{0}=5^{\circ}$

\begin{tabular}{|c|c|c|c|c|c|c|c|c|c|c|c|c|c|c|}
\hline \multirow{2}{*}{ Item } & \multicolumn{14}{|c|}{$\mathrm{C}$ a s e } \\
\hline & 1 & 3 & 5 & 7 & 8 & 9 & 10 & 11 & 12 & 13 & 14 & 16 & 18 & 20 \\
\hline $\begin{array}{r}k_{L}\left[\mathrm{~m}^{-1}\right] \\
\times 10^{-5}\end{array}$ & 0 & 0 & 500 & 320 & 230 & 140 & 50 & 320 & 260 & 200 & 140 & 2 & 140 & 20 \\
\hline $\begin{array}{r}k_{D}\left[\mathrm{~m}^{-1}\right] \\
\times 10^{-5} \\
\end{array}$ & 1,500 & 900 & 1,500 & 1,500 & 1,500 & 1,500 & 1,500 & 1,050 & 1,050 & 1,050 & 1,050 & 1,050 & 600 & 600 \\
\hline$v_{0}[\mathrm{~m} / \mathrm{s}]$ & 1,212 & 1,565 & 1,180 & 1,199 & 1,205 & 1,208 & 1,212 & 1,417 & 1,428 & 1,436 & 1,442 & 1,449 & 1,892 & 1,916 \\
\hline$D[\mathrm{~m}]$ & 266 & 444 & 167 & 265 & 293 & 305 & 293 & 276 & 346 & 396 & 427 & 384 & 631 & 734 \\
\hline$v_{i m p}[\mathrm{~m} / \mathrm{s}]$ & 61.2 & 78.9 & 25.0 & 25.2 & 24.9 & 24.0 & .36 .7 & 30.0 & 30.1 & 30.0 & 29.5 & 70,0 & 39.8 & 58.1 \\
\hline$\varphi_{i m p}\left[{ }^{0}\right]$ & -31.8 & -31.8 & -90.0 & -77.6 & -78.6 & -75.1 & -57.5 & -80.5 & -76.5 & -78.2 & -78.2 & -33.7 & -76.9 & -57.5 \\
\hline$a_{i m p}\left[\mathrm{~m} / \mathrm{s}^{2}\right]$ & 10.9 & 10.9 & 0.2 & 0.1 & 0.6 & 2.0 & 5.2 & 0.2 & 0.1 & 0.2 & 1.0 & 10.3 & 0.0 & 5.2 \\
\hline
\end{tabular}

\section{Conclusion}

The paper presents an original fragmentation model for the identification of the kinematic parameters of the fragments generated by the explosion of a cylindrical tank. The model includes the influence of the temperature manifested through the BLEVE effect. The introduction of initial acceleration is a novelty in the proposed model, which allows a reliable assessment of the velocity of the fragments. Such an approach provides the ability to assess the velocity of a fragment, indicating at the same time that this parameter is not followed by an aleatoric uncertainty. This particularly refers to the direction of the fragment launch (the direction of velocity at the initial moment $-\varphi_{0}$ ), whose values, according to the literature data, follow a uniform distribution. Thus, in the absence of data on the initial launch angle $\varphi_{0}$ of a fragment, the literature assumes a uniform distribution from which its aleatoric uncertainty 
arises (each value of $\varphi_{0}$ has the same probability). However, the fragmentation model shows that the initial launch angle $\varphi_{0}$ depends on the initial acceleration and the aerodynamic characteristics of the fragment. This suggests that the parameter follows the epistemic rather than the aleatoric uncertainty which is found in the literature. This is probably due to the insufficient exploration of the fragmentation model.

The initial acceleration determines the geometric characteristics of the tank $\left(R_{m}, \delta \mathrm{i} \rho\right)$, while the shape of the fragment defines the coefficients of thrust and resistance acceleration $\left(k_{L}\right.$ and $\left.k_{D}\right)$. In the study, the fluctuation effect of air resistance and thrust during the fragment flight is analysed in a unique and original way using the Monte Carlo simulation. Probability density functions for the range of a fragment of a mass $200 \mathrm{~kg}$ are determined. An increase in the launch angle leads to a reduction in the range of the fragment. The maximum range of a fragment of a mass $200 \mathrm{~kg}$ corresponds to the launch angle $\varphi_{0}$ of up to $35^{\circ}$. Due to the pronounced thrust, aerodynamic fragments achieve the maximum range at smaller launch angles. An increase in the launch angle results in a reduction in the range of other constant parameters of the fragment. Trajectories of fragments are a basis for hazard identification and for the assessment of fragmentation risks in the process industry. The trajectory shape defines the ratio of thrust to air resistance. The increase in the fragment thrust and air resistance positively influences fragmentation hazards. The authenticity of the fragmentation analysis implies a probabilistic assessment of the range. Horizontal cylindrical tanks should be placed in the direction of the target in order to reduce potential hazards from the impact of fragments.

\section{REFERENCES}

[1] B.Hemmatian, B. Abdolhamidzadeh, R.M. Dabra, J. Casal, The significance of domino effect in chemical accidents, J. Loss Prev. Process Ind. 29 (2014) 30-38. https://doi.org/10.1016/j.jlp.2014.01.003

[2] B. Abdolhamidzadeh, T. Abbasi, D. Rashtchian, S.A. Abbasi, Domino effect in process industry accidents - An inventory of past events and identification of some patterns, J. Loss Prev. Process Ind. 24 (2011) 575-593. https://doi.org/10.1016/j.jlp.2010.06.013

[3] R.K. Eckhoff, Boiling liquid expanding vapor explosions (BLEVEs): A brief review, J. Loss Prev. Process Ind. 32 (2014) 30. https://doi.org/10.1016/j.jlp.2014.06.008

[4] D. Sun, J. Jiang, M. Zhang, Z. Wang, Influence of the source size on domino effect risk caused by fragments, J. Loss Prev. Process Ind. 35 (2015) 211-223. https://doi.org/10.1016/j.jlp.2015.05.005

[5] N. Khakzad, P. Amyotte, V. Cozzani, G. Reniers, H. Pasman, How to address model uncertainty in the escalation of domino effects?, J. Loss Prev. Process Ind. (2018). https://doi.org/10.1016/j.jlp.2018.03.001

[6] G. Landucci, F. Argenti, G. Spadoni, V. Cozzani, Domino effect frequency assessment: The role of safety barriers, J. Loss Prev. Process Ind. 44 (2016) 706-717. https://doi.org/10.1016/j.jlp.2016.03.006

[7] J. Kang, J. Zhang, J. Gao, Analysis of the safety barrier function: Accidents caused by the failure of safety barriers and quantitative evaulation of their performance, J. Loss Prev. Process Ind. 43 (2016) 361371. https://doi.org/10.1016/j.jlp.2016.06.010

[8] CCPS, Guidelines for Evaluating the Characteristics of Vapor Cloud Explosions, Flash Fires and BLEVE's, Center for Chemical Process Safety, American Institute of Chemical Engineers, New York, 1994. https://doi.org/10.1002/9780470938157

[9] W.E. Baker, J.J. Kulesz, R.E. Ricker, P.S. Bessey Westine, V.B. Parr, Workbook for Predicting Pressure Wave and Fragment Effects of Exploding Propellant Tanks and Gas Storage Vessels. NASA CR-134906. NASA Scientific and Technical Information Office, 1997, Washington.

[10] U. Hauptmanns, A Monte-Carlo based procedure for treating the flight of missiles from tank explosions, Prob. Eng. Mech. 16 (2001) 307. https://doi.org/10.1016/s0266-8920(01)00023-6

[11] U. Hauptmanns, A procedure for analyzing the flight of missiles from explosions of cylindrical vessels, J. Loss Prev. Process Ind. 21 (2001) 395. https://doi.org/10.1016/s0950-4230(01)00011-0

[12] D. Sun, J. Jiang, M. Zhang, Z. Wang, Y. Zang, L. Yan, H. Zhang, X. Du, Y. Zou, Investigation on the approachof intercepting fragments generated by vessel explosion using barrier net, J. Loss Prev. Process Ind. 49 (2017) 989-996. https://doi.org/10.1016/j.jlp.2016.10.012 
[13] H.L. Brode, Blast wave from a spherical charge, Phys. Fluids. 2 (1959) 217-229.

[14] M.R. Baum, The velocity of missiles generated by the disintegration of gas pressurized vessels and pipes. Trans. ASME. 106 (1984) 362-368. https://doi.org/10.1115/1.3264365

[15] A. Mébarki, F. Mercier, Q.B. Nguyen, R.A. Saada, Structural fragments and explosions in industrial facilities. Part I: Probabilistic description of the source terms, J. Loss Prev. Process Ind. 22 (2009) 408416. https://doi.org/10.1016/j.jlp.2009.02.006

[16] A. Tugnoli, G. Gubinelli, G. Landucci, V. Cozzani, Assessment of fragment projection hazard: Probability distributions for the initial direction of fragments, J. Hazard. Mater. 279 (2014) 418-427. https://doi.org/10.1016/j.jhazmat.2014.07.034

[17] G. Gubinelli, S. Zanelli, V. Cozzani, A simplified model for the assessment of the impact probability of fragments, J. Hazard. Mater. A1 16 (2004) 175-187. https://doi.org/10.1016/j.jhazmat.2004.09.002

[18] S. Mannan, Lees' Loss Prevention in the Process Industries, fourth ed., Elsevier, Oxford, 2012.

[19] M.R. Baum, The velocity of large missiles resulting from axial rupture of gas pressurised cylindrical vessels, J. Loss Prev. Process Ind. 14 (2001) 199-203. https://doi.org/10.1016/s0950-4230(00)00039-5

[20] Q.B. Nguyen, A. M. Mébarki, R.A. Saada, F. Mercier. M. Reimeringer, Integrated probabilistic framework for domino effect and risk analysis, J. Loss Prev. Process Ind. 40 (2009) 892-901. https://doi.org/10.1016/j.advengsoft.2009.01.002

[21] G. Gubinelli, V. Cozzani, Assessment of missile hazards: Identificaton of reference fragmentation patterns, J. Hazard. Mater. 163 (2009) 1008 - 1018. https://doi.org/10.1016/j.jhazmat.2008.07.056

[22] EN 13445-3:2014 - Unfired pressure vessels - Part 3: Design, 2014.

[23] EN 13445-5:2014 - Unfired pressure vessels - Part 5: Inspection and testing, 2014.

[24] P. Ciarlet, Mathematical Elasticity, Volume III: Theory of Shells, first ed., Elsevier, 2000.

[25] S.B. Lee at all: A study on the two row effect in the sloshing phenomenon, Transactions of Famena, 38 4 (2014) $29-42$.

[26] D. Royer, E. Dieulesaint, Elastic Waves in Solids I: Free and Guided Propagation, Springer-Verlag Berlin Heidelberg, 2000.

[27] M. Krpan: Dinamika - teorija i primjena, Rijeka 2001. (in Croatian).

Submitted: $\quad 23.6 .2018$

Accepted: $\quad$ 13.3.2019
Goran Tepić

gtepic@uns.ac.rs

Bojan Lalić

blalic@uns.ac.rs

Ilija Tanackov

ilijat@uns.ac.rs

Siniša Sremac

sremacs@uns.ac.rs

Stevan Milisavljević

steva@uns.ac.rs

University of Novi Sad, Faculty of

Technical Sciences

Trg Dositeja Obradovića 6, Novi Sad,

Serbia

Milan Kostelac

milan.kostelac@fsb.hr

University of Zagreb, Faculty of

Mechanical Engineering and Naval

Architecture

Ivana Lučića 5, Zagreb, Croatia 